\title{
PB1-F2 expedition from the whole protein through the domain to aa residue function
}

\author{
I. KOŠÍK ${ }^{1}$, J. HOLLÝ2, G. RUSS ${ }^{1}$ \\ ${ }^{1}$ Institute of Virology, Slovak Academy of Sciences, Dúbravská cesta 9, 84505 Bratislava, Slovak Republic; ${ }^{2}$ Department of \\ Microbiology and Virology, Faculty of Natural Sciences, Comenius University, Bratislava, Slovak Republic
}

\begin{abstract}
Summary. - More than decade ago during systematic search for alternative reading frame derived peptides encoded by influenza A virus recognized by $\mathrm{CD} 8^{+} \mathrm{T}$ cells, $\mathrm{PB} 1-\mathrm{F} 2$ protein was discovered serendipitously by Chen $e t$ al. (2001). Since that time, an increasing body of evidence has continued to highlight the multifunctional meaning of this unusual influenza A protein. After twelve years of intensive research with 56 pubmed records for PB1-F2 in the title there is still a lot yet to explore. Is it a proapoptotic "explosive" protein that suppresses the mechanisms of early innate immune response or does it function as an NS1 antagonist? What is the root of its strain and cell specificity? What is the relationship between PB1-F2 and pathogenicity or secondary bacterial infection? Here we attempt to "take a trip" from the whole protein level through domains and regions to very particular aminoacid residues in correlation with its function in different virus isolates, cell type or animal model.
\end{abstract}

Keywords: PB1-F2; domain; pathogenicity; virulence; influenza A virus

\section{Contents:}

1. Introduction

2. Influenza viruses encode either full length, truncated or no PB1-F2 at all

3. Inflammation, pathogenic effects of PB1-F2 and secondary bacterial infection

3.1 PB1-F2 with residues L62, R75, R79 and L82 display inflammatory activity while PB1-F2 with P62, H75, Q79 and $\mathrm{S} 82$ display antimicrobial activity

3.2 PB1-F2 mutations T51M, V56A and E87G may decrease lethality

3.3 PB1-F2 N66S polymorphism and the complex role of this aa residue

4. PB1-F2 modulates type I interferon and other cytokine responses

E-mail: virukosi@savba.sk; phone:+421-2-59302442.

Abbreviations: IAV = influenza A virus; HK97 = A/Hong Kong/156/1997(H5N1); $1918=$ A/Brevig Mission/1/1918 (H1N1); HK68 = A/Hong Kong/1/1968 (H3N2); VH95 = A/Wuhan/359/1995 (H3N2) non-inflamatory IAV strain; HPAI = highly pathogenic avian influenza virus; LPAI = low pathogenic avian influenza virus; $\mathrm{pdm}=$ pandemic virus; $\mathrm{VDAC}=$ voltage dependent anion channel; ANT $=$ adenosine nucleotide transporter
5. Intracellular localization of PB1-F2 is critical for its ability to enhance vRdRp activity

6. Subcelullar localization of PB1-F2 and its relation to apoptosis

6.1 Leucines, lyzines and arginines aa of mitochondrial targeting sequence

7. PB1-F2 serine 35 and threonine 27 phosphorylation acceptor sites and their role in apoptosis

8. Conclusion

\section{Introduction}

Among human health treating viruses, influenza A virus (IAV) occupies eminent space since few other viruses including HIV or smallpox have been observed to possess such worldwide spreading potential (Fouchier et al., 2012; Moszynski, 2012). ssRNA segmented genome of the IAV code to date 16 protein products, some of which are expressed by alternative splicing (NS2, M2, M3), some by ribosomal frame shift (PB1-F2, N40, PA-X) and some by different ATG in frame translation initiation (PA-N155 and PA-N182) (Muramoto et al., 2012). There are several features predetermining IAV to be such a successful virus. Multiple host species including birds and mammals make 
it impossible for IAV to be globally eradicated. Antigenic shift and drift as the major driving force of IAV variability enable virus to overcome pre-existing immunity in population mediated mainly by virus neutralizing antibody (Hoper et al., 2012). The "hit and run" strategy of rapid replication and spreading to further victims ensures that host immune system is not capable of establishing an antiviral state soon enough to hinder/block infection. Another important factor affecting virus long term spreading is its ability to suppress immune response (Friesenhagen et al., 2012), virus replication kinetics (Hatta et al., 2001; Ozawa et al., 2011; Smith et al., 2011) and cleavability of HA glycoprotein (Kido et al., 2012). The aforementioned IAV features resemble the overall devastating effects on host organisms termed pathogenesis. Multiple proteins of IAV have been shown to contribute to the pathogenetic effects of influenza A virus including IFN antagonist NS1 (Ehrhardt et al., 2010). HA possessing multibasic cleavage site (Kido et al., 2012) or M2 and its relation to inflamasome induction (Ichinohe et al., 2010).

PB1-F2 discovered by Chen et al. (2001) represents another widely accepted viral factor of pathogenicity. This small protein with a molecular weight of $10,5 \mathrm{kDa}$ was shown to target mitochondrial membrane (Gibbs et al., 2003; Yamada et al., 2004), to induce cell death via interaction with VDAC3 and ANT1 mitochondrial membrane proteins (Zamarin et al., 2005), impact virulence and pathogenesis in mouse model (Zamarin et al., 2006) by disrupting alveolar macrophages
(Coleman, 2007) and increase secondary bacterial infection (McAuley et al., 2007). It has been shown more recently that single mutation N66S contribute to increased virulence of HPAI H5N1 (HK97) and 1918 IAV (Conenello et al., 2007). It is not yet clear what the pathogenicity determinants of the PB1-F2 are but it is probable that interaction with viral polymerase subunit PB1 (Kosik et al., 2011; Mazur et al., 2008) affecting catalytic activity contributes to the pathogenicity of IAV. Despite some discrepancy surrounded in relation to IFN type I response (Le Goffic et al., 2010), it is reasonable to think that PB1-F2 plays an important role in IFN type I response modulation (Conenello et al., 2011; Dudek et al., 2011; Le Goffic et al., 2010; Varga et al., 2011). Sequence variability of PB1-F2 from different strain mirrors distinct structural signatures (Solbak et al., 2012). Sequence alignment of the PB1 proteins of five distinct viruses (ABA55039 A/Brevig Mission/1/1918(H1N1), NP_040985 A/Puerto Rico/8/1934(H1N1), ACU79879 A/Hong Kong/1/1968(H3N2), YP_308665 A/Goose/ Guangdong/1/96(H5N1), ADA72001 A/swine/4/ Mexico/2009(H1N1)) revealed that there was a $92,5 \%$ sequence position identity while alignment of the PB1-F2 proteins of the same viral isolates showed just $39,6 \%$ sequence position identity (Fig. 1). This discrepancy is caused by degeneracy of the genetic code as third nucleotides of PB1 codons equals second nucleotides of PB1-F2 codons. Since PB1-F2 is one of the most variable IAV proteins it is not surprising

(a)

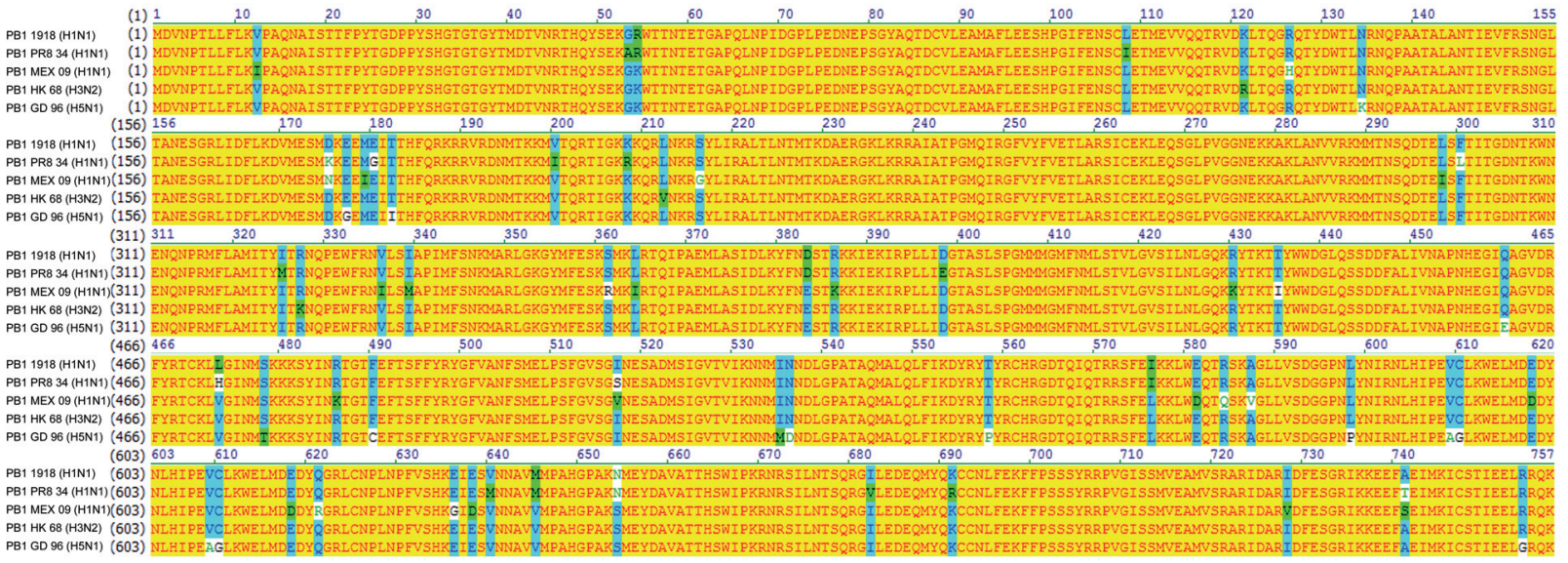

(b)

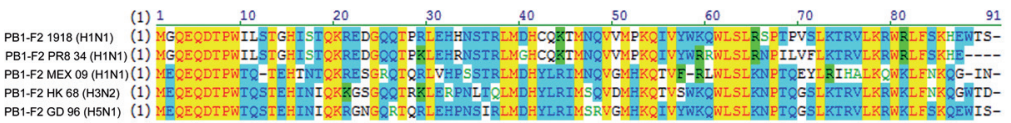

Fig. 1

VNTI advanced 11.5.0 based alignment of the PB1 and PB1-F2 aminoacid sequences of the five distinct IAV isolates

a) PB1 proteins of the A/Brevig Mission/1/1918(H1N1) [1918], A/Puerto Rico/8/1934(H1N1) [PR8 34], A/Hong Kong/1/1968(H3N2) [HK68], A/Goose/ Guangdong/1/96(H5N1) [GD96], A/swine/4/Mexico/2009(H1N1) [MEX 09]. b) PB1-F2 proteins of the identical isolates shown variability of the PB1-F2 $\mathrm{ORF}$ and a high degree of conservancy of the PB1 protein sharing the same RNA segment. Identical aa displayed yellow-red, synonymous green-black, prevalent blue-blue, minor-non-synonymous black color. 
that the effects of the PB1-F2 are strain, cell type and host specific (Hai et al., 2010; Chen et al., 2010; Marjuki et al., 2010; McAuley et al., 2010a,b; Pena et al., 2012a; Schmolke et al., 2011) During the last pandemics in 2009 it was speculated that the introduction of the stop codons in the PB1-F2 ORF after 11 aa residue could be responsible for the mild course of infection (Schnitzler and Schnitzler, 2009). Subsequently, several groups showed the mild effect of reconstitution of the PB1-F2 ORF on virulence and pathogenicity of pdm H1N1 2009 flu (Hai et al., 2010; Ozawa et al., 2011; Pena et al., 2012b). The overall importance of the PB1-F2 for virus seems to be very complex, influenced by multiple factors including its aminoacid sequence variability, other viral protein cooperation, host cell protein interactions as well as host species background. Here we provide a global overview on the role of PB1-F2 at different structural levels including whole protein, domain, region and very particular amino acids residues in relation to the above mentioned properties.

\section{Influenza viruses encode either full length, truncated or no PB1-F2 at all}

PB1-F2 represents a very unstable protein since proteasome has been shown to be involved in its rapid degradation (Chen et al., 2001; Schmolke et al., 2011). Degradation is probably one of the most important properties of the PB1F2 as the level of the protein in general affects its functions. Expression is affected by a complex mode of infection. During in vitro infection of the A549 epithelial cell line with A/ WSN/33 H1N1, PB1-F2 is detectable first in 6HPI, and in 24HPI it was not detectable at all (Le Goffic et al., 2010). Interestingly, under natural infection in vivo 48HPI is first detected in PB1-F2 in lung tissue. Later post infection from A/WSN/33 72HPI and 96HPI it was still possible to detect $\mathrm{PB} 1-\mathrm{F} 2$ expressed by the same strain A/WSN/33 in the lungs of C57/BL/6 mice (Le Goffic et al., 2011). It is important to note that the inability to detect PB1-F2 could be caused by the formation of amyloid fibers (Chevalier et al., 2010). Comparison of the growth kinetics between recombinant virus USSR/90/77 expressing C-terminally truncated form of the PB1-F2 protein 57aa long and it's PB1-F2 deletion variant has shown that despite minor differences, a delay in PB1-F2 deficient virus growth using MDCK cell line for titration was observed. Growth properties of another recombinant virus based on the same virus background expressing N66S PB1-F2 gene from 1918 Spanish flu virus did not differ significantly in comparison with C-terminally truncated PB1F2 expressing virus. (Meunier and von Messling, 2012). In agreement with our observations, $\mathrm{N}$ terminus is responsible for increased PB1 and other viral protein expression (Kosik et al., 2011) as well as overall replication kinetics (Smith et al., 2011).Such observation of MDCK cell is important since this cell line has been shown to replicate IAV very efficiently (Ueda et al., 2008) and the contribution of PB1-F2 to replication kinetics is more significantly visible in the A549 cell line. Reconstitution of the PB1-F2 from the pdm H1N1 2009 IAV on the background of PR8 elevated virus growth in comparison to 11aa WT PB1-F2 from the same isolate. Interestingly, despite truncated PB1-F2 had produced twice as large plaques than full length recombinant whereas application of the same PFU on the cells had produced almost twice the plaque count after full length PB1-F2 virus infection (Chen et al., 2010). As both of the viruses possess the same HA, equal adsorption on infected cell surface is probable. Thus an increased plaque count suggests more effective defence against cell antiviral response (IFN type I) to be established in full length PB1-F2 pdm H1N1 2009 expressing virus infected cells. Using ex vivo ferret and macaque, lung cultures displayed no differences in replication kinetics 24 and 48, neither for HPI USSR/90/77 PB1-F2 (57aa) nor PB1-F2 knock out for PB1-F2 1918 viruses. On the other hand, PB1-F2 1918 expressing recombinant virus developed more severe clinical symptoms in ferret model (Meunier and von Messling, 2012). In another epidemiologically important host swine, infection with A/California/04/09 with restored ORF of full length PB1-F2 resulted in increased replication and histopathology in the infected nasal turbinates, trachea and proximal lung 24HPI (Pena et al., 2012b). Similar effects were observed ex as well as in vivo. Common reservoir of the IAV is generally accepted by waterfowl. Since more than $95 \%$ of avian IAV isolates preserved PB1-F2 ORF 90 aa long it is reasonable to think that $\mathrm{PB} 1-\mathrm{F} 2$ plays an important role in this species. In agreement with this hypothesis, deletion of PB1-F2 ORF from HPAI H5N1 slows progression of clinical signs and sickness and even leads to decreased mortality in comparison with $\mathrm{PB} 1-\mathrm{F} 2$ expressing virus. $\mathrm{PB} 1-\mathrm{F} 2$ seems to play a role in organ dissemination as its expression leads to increased viral titers in spleen, colon, lung, liver and kidney 1DPI. Later post infection viral titers are equal, suggesting the early infection phase role of PB1-F2 in ducks. Deletion or N66S polymorphism of the PB1-F2 has no effect on HPAI to LPAI flip (Schmolke et al., 2011).

\section{Inflammation, pathogenic effects of PB1-F2 and secondary bacterial infection}

Inflammation of IAV infected tissue is an important factor of pathogenicity for example in the case of 1918 IAV or HK97 (Alymova et al., 2011; Conenello et al., 2007). Pathogenicity of the IAV could be divided into virus determined and host immune system determined. Overall pathogenesis of the IAV is a consequence of the cooperation of the aforementioned factors. PB1-F2 possesses both of these factors. Its relation to virus RNA dependent RNA polymerase (vRdRp) fits the cri- 
teria for virus determined pathogenicity contribution (Kosik et al., 2011; Mazur et al., 2008; McAuley et al., 2010b). In the terms of host immune system contribution, modulation of the cytokines response, selective immune cell pro-apoptosis (Coleman, 2007) and more recently identified neutrophil chemoattraction has been described for PB1-F2 functions (Conenello et al., 2011; Le Goffic et al., 2011). Capacity to enhance the inflammatory response seems to be a general feature of $\mathrm{PB} 1-\mathrm{F} 2$ proteins encoded by $\mathrm{PB} 1$ genes that are direct introductions from the avian gene pool. Notably, PB1 segment has been newly introduced for all pandemics of the $20^{\text {th }}$ century. Exposition of human lung epithelial cells A549 or mouse macrophage cell line RAW264.7 to panel of the C-terminal peptides of the PB1-F2 caused cell death only in the case of PR8 and 1918 viruses for RAW264.7 and PR8 for A549 cell line respectively. No significant cell death was observed for any other H2N2 1957, H3N2 1968, H3N2 1995, and H5N1 2004 PB1-F2 derived C-terminal peptides. Exposition of mice lungs to the same panel of peptides caused an increased dentritic cells, T cell, macrophage and neutrophil count for all subjected peptides (McAuley et al., 2010a). Similarly increased bronchoalveolar cellularity was observed in another study with HK97 S66 PB1-F2 variant (Conenello et al., 2011). An infection study with recombinant viruses expressing various PB1-F2 confirmed peptide based observations for PR8 and 1918 PB1-F2 which are the only ones with cell death induction potential. One difference is that while cell death induction capable peptides derived from PB1-F2 of the PR8 and 1918 act on the RAW264.7 cell line only, infection with PR8 viruses expressing PB1-F2 or the PR8 and 1918 induced cell death solely on theA549 cell line. In general, PR8 PB1-F2 is the most potent cell death inductor. This observation means that cell death is a strain specific factor, which does not affect pathogenesis of the IAV in common (McAuley et al., 2010a). High throughput PB1-F2 interaction screening of human leukocyte cDNA library in two hybrid systems revealed macrophage migration inhibitory factor (MIF) as an interaction partner of the PB1-F2 (Guan et al., 2012). Since MIF is responsible for the preservation of inflammatory states by preventing untimely apoptosis of the macrophages and monocytes during infection, this interaction could affect functions of this multitropic cytokine (Mitchell et al., 2002) and mediate pro-inflammatory function of the PB1-F2 during IAV infection by recently unknown mechanism.

3.1 PB1-F2 with residues L62, R75, R79 and L82 display inflammatory activity while PB1-F2 with P62, H75, Q79 and S82 display antimicrobial activity

H3N2 viruses have evolutionary expressed full length PB1-F2 with inflammatory (A/Hong Kong/1/1968 (HK68)) or its non-inflammatory descendant (A/Wuhan/359/1995
(WH95)). PB1-F2 from these two related viruses differ in 5 aa of the C-terminal part (61 to 90 aa) of the molecule. Administration of 26aa long peptide variants with altered aminoacids in $62,75,79$, and 82 to mice lung managed to completely revert pro/non-inflammatory phenotype measured as body weight change and mortality. The most significant effect was observed in the case of HK68 derived peptide and quadruple mutation WH95 L62,R75, R79, L82 which was characterized by increased TNF- $\alpha$ level, extensive lung consolidation with dense neutrophil monocyte and macrophage infiltrates. Interestingly, while secondary infection with S. pneumonie was increased 70 fold following treatment of mice with $\mathrm{HK} 68 \mathrm{H} 3 \mathrm{~N} 2$ derived pro-inflammatory peptide in comparison to PBS treated group, titre of bacteria was significantly lower in the group of mice treated with WH95 non-inflammatory peptide in comparison to the negative control group suggesting the antibacterial potential of P62, H75, Q79, and S82 conferring peptide. The bactericidal effect of these residues was confirmed by exposing $S$. pneumonie to non-inflammatory peptide derived from WH95 virus (Alymova et al., 2011).

\subsection{PB1-F2 mutations T51M, V56A and E87G may} decrease lethality

These tree mutations in PB1-F2 ORF of the HPAI H5N1 A/Vietnam/1203/2004 have been established to decrease in lethality in mallard ducks. Despite the fact that viruses replicate in mallard ducks to a similar 3DPI level regardless of aminoacids in 51, 56 or 87 positions respectively, primer extension assay clearly displayed that $\mathrm{T}, \mathrm{V}$ and $\mathrm{E}$ aminoacids in C-terminal end of the PB1-F2 increase accumulation of viral RNA during the infection cycle. Additionally, these aminoacids affect the activity of vRdRp (Marjuki et al., 2010).

\subsection{PB1-F2 N66S polymorphism and the complex role of} this a a residue

The rare polymorphism of PB1-F2 in position 66 was described as a factor affecting virulence and pathogenicity of IAV. Serine in this position was identified in 1918 IAV and HPAI HK97 to increase pathogenicity in mouse model. Recombinant virus expressing S66 replicated 100-fold higher than N66 isogenic variant (Conenello et al., 2007). Expression of the HK97derived S66 form of PB1-F2 in mouse lung epithelial adenoma cell line (LA-4) 24HPI was almost undetectable in comparison to infection with isogenic virus with PB1-F2 N66. It is surprising that despite inability to detect PB1-F2 S66, such virus exhibit 10-fold higher virus titre on LA-4 cell line than WT virus expressing a high level of PB1F2 24HPI (Schmolke et al., 2011). This is in agreement with a report by Varga et al. (2011) which showed decreased IFN- $\beta$ induction despite a lower amount of S66 PB1-F2 PR8 being 
produced. Neither WT nor S66 variant of PB1-F2 co-localize with inner mitochondrial membrane protein prohibitin, but rather diffuse cytoplasm and nucleus distribution. In mouse model C57/BL/6/A2G-Mx1, expressing Mx1 protein it was shown that S66 PB1-F2 mutation increase 10-fold $\mathrm{LD}_{50}$ in comparison to N66 PB1-F2 variant. Neurological symptoms were observed for S66 PB1-F2 from the HK97 in mice model while deletion mutant and N66 WT form of the PB1-F2 did not exhibit such features. Additionally, infection of only S66 PB1-F2 expressing virus had spread to the brain 8DPI in 6 of 7 C57/BL/6/A2G-Mx1 mice, while no virus was detected in the spleen or lung on day 8 post infection. Given that LPAI H5N1 lacking HA multibasic cleavage site does not differ in lethality regardless of aminoacid in position 66 of the PB1F2 suggests that tissue tropism determines the impact of the PB1-F2 (Schmolke et al., 2011). In duck fibroblast cell line S66 variant of the PB1-F2 is expressed in a lower amount than N66 form. The serine variant had slightly enhanced disease progression in ducks. The course of infection with S66 or N66 PB1-F2 isogenic viruses A/WSN/33 with PB1 segment derived from $\mathrm{HK} 97$ in transgenic adaptive immune system deficient mouse model (RAG1-/-, CD4-/-, CD8-/- or IFN- $\gamma-/-)$ compared to infection of the WT mouse model exhibits similar decreased body weight (0 to 7/11DPI) in S66 PB1-F2 virus. Authors had concluded none of the $\mathrm{CD}^{+}$, $\mathrm{CD}^{+}$or $\mathrm{B}$ cell to be essential for pathogenesis mechanism (Conenello et al., 2011). Since virus specific $\mathrm{CD}^{+}$cells are initially detectable 5DPI with peaks between 9,5DPI to $11 \mathrm{DPI}$ and IgM serum level peaks 8DPI to $10 \mathrm{DPI}$ while virus titre peaks 2 to 3DPI, with complete viral clearance by day 11 (Miao et al., 2010), it is not surprising that adaptive response did not affect morbidity in these experiments. However it would be interesting to study PB1-F2 in the same experimental mice models with secondary infection by homologous and heterologous viruses. Transcriptional profiling of mouse lungs infected with S66 and N66 PB1-F2 HK97 have clearly shown that S66 variant induces a lower level of the RIG-1, IFN- $\beta, \mathrm{Mx} 1$, and STAT- 1 1DPI, but higher levels of IFN regulated response 3DPI in comparison with N66 PB1-F2. Thus serine 66 in PB1-F2 protein of the HK97 caused a delay in early interferon response in mice (Conenello et al., 2011). Uncontrolled virus replication during the first days post infection may result in an increased count of infected lung cells which in turn could cause higher cytokine production and increased cellularity of immune cells to lungs. This hypothesis is supported by increased levels of multiple cytokines in lungs 3DPI and later post infection including IFN- $\beta$, IL-6, KC (monocyte trafficking to lungs), M-CSF (macrophage activation and maturation), TNF- $\alpha$, MCP-1, RANTES, IFN- $\gamma$. Increased monocytes, neutrophils, and dentritic cells have also been reported for S66 PB1-F2 of the HK97 (Conenello et al., 2011). Similar observations were made for reconstituted S66 PB1-F2 from pdm 2009 H1N1 strain (Hai et al., 2010). As effects of PB1-F2 variants differ significantly in relation to time post infection, it would be interesting to compare kinetics of the PB1-F2 expression to kinetics of expression of the IFN- $\beta$,- $\alpha$ and the other cytokines. Such an experiment could contribute to resolving the PB1-F2 $\leftrightarrow$ cytokines enigma. Despite PB1-F2 seemingly inhibiting IFN- $\beta$ induction in MAVS dependent manner (Varga et al., 2011), the additional indirect impact of the PB1-F2 on cytokine expression should not be excluded. Increased expression of the NS1 and other viral proteins in the presence of the N-terminal part of PB1-F2 (Kosik et al., 2011; Mazur et al., 2008) as well as the virus co-infection dependent effect of the PB1-F2 on cytokines (Le Goffic et al., 2010) support this assumption. IFN- $\beta$ antagonism seems to be strain independent and S66 enhanced. PR8 derived PB1-F2 protein with N66S mutation exhibit fold increased IFN-I inhibition compared to WT PB1-F2. Additionally, enhanced IFN-I suppression in murine lungs epithelial cell line LA- 4 as well as murine and human dentritic cells was also observed supporting IFN antagonism of the PB1-F2 as general feature (Varga et al., 2011). Reconstitution of the PB1-F2 ORF in pdm H1N1 2009 with serine in 66 position leads to higher viral titers on A549 human lung epithelial cells than N66 variant. However both of these viruses produced similar size plaques on MDCK cell line. In the Balb/c mouse model there was no difference in replication kinetics of S66 or N66 PB1-F2 expressing viruses. In the DBA/2 mouse model, both viruses replicated to a similar level (Hai et al., 2010). It has recently been shown that $\mathrm{DBA} / 2$ mice are more susceptible to IAV infection than Balb/c (Boon et al., 2009). Thus overall effects of PB1-F2 might be overplayed. Secondary bacterial infection of the $S$. pneumonie revealed a 5-fold increase of this respiratory pathogen in the group infected with S66 PB1-F2 IAV in comparison to 11aa PB1-F2 expressing IAV. N66 PB1-F2 IAV caused a 2-fold increase in S. pneumonie titer. Thus neither PB1-F2 from pdm H1N1 2009 nor S66, N66 variants of the PB1-F2 significantly affect secondary bacterial infection. In ferrets, only slightly more weight loss with similar virus titers in nasal wash was associated with S66 PB1-F2 expression in comparison with N66 or 11aa ORF expressing IAV (Hai et al., 2010).

\section{PB1-F2 modulates type I interferon and other cytokine responses}

To restrict virus proliferation, IAV-infected cells mount a strong antiviral response. IFN- $\beta$ is the most potent antiviral cytokine and is massively produced during IAV infection after pathogen associated molecule recognition by TLR3, RIG-1, NLR, and MDA-5 (Guillot et al., 2005; Le Goffic et al., 2007; Varga et al., 2011). Upon recognition of viral RNA species, RIG-1 interacts with mitochondrial antiviral protein 
(MAVS also known as VISA, CARDIF or IPS-1). This leads to secretion of IFN and auto/paracrine activation of JAK/ STAT pathway which in turn leads to the formation of ISG3 transcription complex formation and expression of protein kinase R (PKR), Mx GTPase and other antiviral proteins (Varga et al., 2011). In the human epithelial cell line A549, infection with IAV $\triangle$ PB1-F2 caused reduced IFN- $\beta$ level $24 \mathrm{HPI}$, while $8 \mathrm{HPI}$ showed no difference in IFN- $\beta$ level regardless of the presence or absence of the PB1-F2 suggesting that PB1-F2 of amyloid fibrils can exacerbate IFN- $\beta$ response. This effect seems to be restricted to epithelial cell line as infection of immune cell line Jurkat, U937 or mouse alveolar macrophages induces a similar level of IFN- $\beta$ in response to $\mathrm{PB} 1-\mathrm{F} 2$ presence or absence. Importantly, induction of IFN- $\beta$ by PB1-F2 from several distinct IAV strains is fully dependent on co-infection with $\triangle \mathrm{PB} 1-\mathrm{F} 2$ virus (Le Goffic et al., 2010). Thus PB1-F2, not solely but probably in cooperation with other IAV expressed proteins contribute to IFN- $\beta$ response during the late stage of epithelial cell line IAV infection. IAV viruses with alteration in ATG start codon of the PB1-F2 could express N-terminally truncated form of the $\mathrm{PB} 1$ protein termed N40 because of PB1 and PB1-F2 share the same genome segment but not the same ORF (Wise et al., 2009). However this protein was shown to have no effect on IFN- $\beta$ (Le Goffic et al., 2011), thus proving that $\mathrm{PB} 1-\mathrm{F} 2$ protein along with $\mathrm{PB} 1, \mathrm{~PB} 2, \mathrm{PA}$ (Graef et al., 2010; Iwai et al., 2010), and NS1 is involved in the IFN- $\beta$ response modulation. The same scientific group has shown induction of the NF- $\mathrm{BB}$, major pro-inflammatory transcription factor, during A/WSN/33 H1N1 infection. Additionally, while higher infection dose exhibits no differences, lower infection dose $\left(5 \times 10^{4} \mathrm{PFU}\right)$ of the Balb/c mice with A/WSN/33 H1N1 WT or $\triangle \mathrm{PB} 1-\mathrm{F} 2$ differs in IFN- $\beta$ induction. WT IAV induced a higher level of IFN $\beta$ mRNA. Detection of pro-inflammatory cytokines IFN- $\gamma$, IL-6, CXCL1/ $\mathrm{KC}$ (major neutrophil chemoattractant) in bronchoalveolar fluid by ELISA revealed that infection of $\mathrm{Balb} / \mathrm{c}$ mice with A/WSN/33 H1N1 virus induces a higher amount of these pro-inflammatory molecules than $\triangle \mathrm{PB} 1-\mathrm{F} 2$ virus (Le Goffic et al., 2011). This suggests a pro-inflammatory role and potential immunopathology effects of PB1-F2. Importantly, virus independent treatment of J774 macrophage cell line with 26aa long peptides derived from HK68 H3N2 PB1-F2 resulted in a 20 -fold increase of TNF- $\alpha$ secretion which was shown for mice lung infected with HK97 H5N1 as well (Conenello et al., 2011), while reconstituted PB1-F2 from pdm 2009 H1N1 did not alter TNF-a level at all (Hai et al., 2010). However, the involvement of $C$-terminal aminoacids L62, R75, R79 and L82 responsible for increased inflammation and TNF- $\alpha$ level (Alymova et al., 2011) post infection by HK68 H3N2 were recently identified. Reconstituted PB1-F2 from pdm $2009 \mathrm{H} 1 \mathrm{~N} 1$ harbours non-inflammatory residues $(\mathrm{P}, \mathrm{H}, \mathrm{Q}$,$) in positions 62,75,79$ identical to non-inflammato- ry HK68 H3N2 descendant WH95. In contrast to Le Goffic studies (Le Goffic et al., 2010; Le Goffic et al., 2011) other groups have shown the opposite function of the PB1-F2 on IFN- $\beta$. In the lung explantates of ferrets and macaques it was established that infection with USSR/90/77 PB1-F2 (57aa) or PB1-F2 knock out or PB1-F2 1918 viruses 24HPI and 48 HPI slightly upregulates IFN- $\beta$, IL- 6 , and IL- 8 cytokines with lower induction by USSR/90/77 PB1-F2 (57aa). Similar experiments on ferret blood derived macrophages resulted in mild and delayed IL- $1 \beta$, IL-6, and IL- 8 mRNA transcription in the case of USSR/90/77 PB1-F2 (57aa) or USSR/90/77 PB1-F2 1918 while PB1-F2 lacking virus infection resulted in upregulation of mentioned cytokines (Meunier and von Messling, 2012). This observation could suggest that the $\mathrm{N}$-terminal half of the PB1-F2 is responsible for the suppression of pro-inflammatory cytokines transcription in immune competent cells. Despite Varga et al. (2011) having shown that the C-terminal domain of PR8 derived PB1-F2 mediates IFN antagonism activity, C-terminal domain in the noted study includes aa fragment corresponding to 38 to $87 \mathrm{aa}$. Overlapping peptide 19 aa long could be involved in IFN modulation activity. In ferret nasal washes of animals infected with USSR/90/77 PB1-F2 1918, the level of IFN- $\alpha$ mRNA 1DPI was 10-fold lower than USSR/90/77 PB1-F2 (57aa) or PB1-F2 knock out viruses while there were no differences in IFN- $\beta$ mRNA level suggesting that the Cterminal part of the PB1-F2 1918 could also influence type I IFN response. Early after infection of pigs with recombinant pdm $2009 \mathrm{H} 1 \mathrm{~N} 1$ virus conferring complete PB1-F2 ORF (A/ California/04/09 PB1-F2), a higher level of IFN- $\alpha$ and IL-1 $\beta$ was induced (Pena et al., 2012b). Infection of the mouse epithelial cell line LA-4 as well as bone marrow derived macrophages and dentritic cell with HPAI HK97 PB1-F2 N66/S66 viruses did not significantly alter the mRNA level of type I and II IFN. However absence of the PB1-F2 leads to higher expression of the IL-1 $\beta$ and IL-6. S66 PB1-F2 induces the lowest level of IFN induced response of all (Schmolke et al., 2011). Reconstituted PB1-F2 derived from pdm 2009 H1N1 strain of the IAV induces a similar level of several cytokines including IFN- $\gamma$ (type II IFN), IL- $1 \beta$, and TNF- $\alpha$ (pdm H1N1 2009 PB1-F2 contains non-inflammatory P,H, $\mathrm{Q}$ motive mentioned above) in Balb/c mouse model while MCP-1, MIP- $1 \beta$ and RANTES were significantly higher in mouse infected with S66 PB1-F2 IAV. In the DBA/2 mouse model S66, PB1-F2 induces no detectable level of IL-1 $\beta$, while RANTES and MIP-1 $\beta$ (the same as for Balb/c) are increased in comparison to 11aa long PB1-F2 in pdm 2009 H1N1 (Hai et al., 2010). Effects outlined of the PB1-F2 are strain specific, however interferon suppression seems to be a common property as H5N1 HK97 as well as PR8 strain both exhibit IFN- $\beta$ antagonism (Varga et al., 2011). On the other hand, host cell factors could even revert this feature since PB1-F2 in A549 human lung epithelial cells, ferrets and 
macaques lung explantates have been shown to exacerbate IFN- $\beta$ and other cytokines levels (Le Goffic et al., 2010, 2011; Meunier and von Messling, 2012).

\section{Intracellular localization of PB1-F2 is critical for its ability to enhance vRdRp activity}

Usually, minigenome polymerase reconstitution system contains in addition to expression plasmids for PB1, PB2, $\mathrm{PA}$, and NP, an extra plasmid for PB1-F2. The rationale for this lies in basal differences in PB1-F2 translation initiation between viral segment based and plasmid based PB1-F2 expression. While Kozak sequences surrounding PB1 in IAV RNA segment are suboptimal, favouring Kozak optimal PB1-F2 translation initiation, in plasmid based expression PB1 ATG is surrounded by optimal Kozak sequences inevitably leading to the favouring of translation of $\mathrm{PB} 1$ protein. In our previous work concerning DNA vaccination with PB1 expressing plasmid, we were not able to detect expression of the PB1-F2 after transfection with PB1 expressing plasmid. Additionally, while we were able to detect anti PB1 antibodies in sera of pPB1 DNA vaccinated mice, we were not able to detect anti PB1-F2 antibodies in the same sera suggesting none or trace expression of PB1-F2 from PB1 expression plasmid (Kosik et al., 2012). Pena et al. (2012b) used the vRdRp activity luciferase reporter system employing expressing plasmid derived from the A/California/04/09 in which PB1 gene contains PB1-F2 ORF. Even employing PB1-F2 expression thereby disadvantaging the reporter system revealed slightly increased the activity of vRdRp (Pena et al., 2012b). It is a matter of speculation as to whether or not independent plasmid for PB1-F2 could provide even higher effects on vRdRp. Furthermore, infection of luciferase reporter plasmid transfected cells with the same virus confirmed the increased activity of vRdRp in the functional PB1-F2 ORF environment. Tree aminoacid changes in HPAI H5N1 A/Vietnam/1203/2004 PB1-F2 namely T51M, V56A, and E87G lead to decreased activity of the vRdRp in chicken fibroblast DF-1 or human 293T cell line. Similar to Pena et al. (2012), Marjuki et al. (2010), and McAuley et al. (2010) used pPB1-F2 less luciferase reporter system. M51, A56, and G87 caused a $20-50 \%$ decrease in polymerase activity (Marjuki et al., 2010). The author's conclusion is in contrast with the results of our group identifying the N-terminal half of the $\mathrm{PB} 1-\mathrm{F} 2$ as the major mediator of $\mathrm{PB} 1-\mathrm{F} 2$ to $\mathrm{PB} 1$ relation (Kosik et al., 2011). However the minor role of the $\mathrm{C}$ terminal part of PB1-F2 cannot be excluded since PB1-F2 effects on vRdRp and the replication cycle could be a multistep process involving several PB1-F2 regions in ordered sequence. Another report confirmed the importance of PB1-F2 for vRdRp activity where stop mutation preventing expression of PB1-F2 had decreased polymerase activity by nearly $35 \%$ in the 293T cells transfected with reporter system harbouring HPAI HK97 derived PB1-F2 (Chen et al., 2010). A similar effect was observed for another H5N1 derived PB1-F2 from A/ Vietnam/1203/04 where blocking PB1-F2 expression led to a more than $50 \%$ decrease of polymerase activity measured in 293T as well as A549 cell lines (McAuley et al., 2010b). In the study mentioned, the effect was strain and cell type dependent since absence of PB1-F2 during infection of PR8 $\triangle \mathrm{PB} 1-\mathrm{F} 2$ had even increased activity of vRdRp on A549 cell line while vRdRp activity was decreased on 293T cells in comparison with WT PB1-F2 expressing virus. WH95 derived PB1-F2 has no measurable effect on vRdRp activity. Subcellular localization preference of the PB1-F2 might dramatically affect functional output of this multifunctional protein. There are at least two possible scenarios for PB1-F2 behaviour during the infection cycle. One is for cytosol and nuclear targeted PB1-F2 affecting most probably the activity of vRdRp. The second is for mitochondrial targeted PB1-F2 thus modifying cytokines response (MAVS) and pro-apoptosis effects. The rate of one pathway to a second should then be determined by PB1-F2 aminoacid composition and host cell factors available in situ at the time. This hypothesis of the "disbalanced" behavior of PB1-F2 is in agreement with the observation that viruses expressing PB1-F2 with suboptimal MTS are prevalently localized to cytoplasm and nucleus and their mitochondrial targeting sequence (MTS) optimal mutants with increased mitochondrial localization have dramatically decreased vRdRp activity (Chen et al., 2010).

\section{Subcelullar localization of PB1-F2 and its relation to apoptosis}

A pioneering report describing PB1-F2 discovery outlined mitochondrial localization of the PB1-F2 protein (Chen et al., 2001), which was confirmed by taq fusion studies identifying MTS to region 69 to 82 as minimal MTS and 65 to 87 as optimal MTS (Gibbs et al., 2003). Some discrepancies surrounded precise mapping of MTS since using different a fusion flag resulted in the identification of 46 to 75 as minimal MTS. In the case of the infection of mouse lung epithelial cells LA-4 and the infection of duck fibroblasts PB1-F2 derived from HPAI HK97 localized mainly to the cytoplasm and nucleus, no significant co-localization with mitochondrial membrane protein prohibitin is displayed regardless of the presence or absence of serine residue in position 66 (Schmolke et al., 2011). Comparison of subcellular localization of the different strain derived PB1-F2 exhibit variation. PB1-F2 from A/Taiwan/3355/1997 H1N1 and commonly used laboratory strain PR8 share subcellular distribution with mitochondrial preferences despite more than six decades of evolution. Surprisingly, PB1-F2 derived from the HK97 and H7N7 A/Netherlands/219/2003 does not 
specifically localize to mitochondria, but a cytoplasmic and nuclear pattern is typical for this isolate. Since most protein variation is localized to C-terminal domain as well as minimal mitochondrial targeting sequence (Chen et al., 2010), it is not surprising given the variability of mitochondrial localization. The purpose of such inconsistency most probably lies in the advantage of nonessential nature of the PB1-F2 for IAV. Observed cell, strain and host determined feature variability of $\mathrm{PB} 1-\mathrm{F} 2$ could provide a crucial advantage to the virus during novel host adaptation. Thus while in avian host it might be more important for PB1-F2 to function in nucleus as observed for HK97 PB1-F2, in mammalian host mitochondrial localization with pro-apoptosis effects on immune cells might be desired as in the case of PR8 PB1-F2. This is in agreement with no effect of HK97 PB1-F2 expression having been observed at the apoptosis induction level on human monocytic cell line U937, but increased vRdRp activity and virus growth (Chen et al., 2010). Recently discovered interaction of PB1-F2 with MIF protein (Guan et al., 2012) will hopefully bring some light to relation to PB1-F2 and apoptosis as MIF prevents apoptosis in macrophage cells (Mitchell et al., 2002) thus preserving inflammation. It will be interesting to compare the conservancy and presence of MIF interaction responsible aminoacid residues of the different strain derived PB1-F2 with its ability to induce apoptosis in immune competent cells.

\subsection{Leucines, lyzines, and arginines aa of mitochondrial targeting sequence}

Effective mitochondrial targeting has been shown not to be restricted to defined aa in defined position, but rather formation of amphipathic helix determines the ability of PB1-F2 and other virus proteins (HTLV-I p13) to target mitochondrion (Gibbs et al., 2003; Chen et al., 2010; Yamada et al., 2004). Leucines, lysines and arginines play cardinal roles in amphipathic helix formation. Moreover, leucine rich domains were considered to be responsible for interaction with hydrophobic binding groove on TOM20 import receptor on mitochondrial membrane (Pfanner, 2000). Comparison of the PB1-F2 protein sequence from PR8 (predominantly localized to mitochondria) and HK97 (diffusely localized to cytoplasm and nucleus) revealed that while only three $\mathrm{L}$ in positions 72, 77, and 82 are presented in HK97 PB1-F2, four $\mathrm{L}$ residues are presented in positions $69,72,78$, and 82 of the PB1-F2 from the PR8 virus (Chen et al., 2010). Both of these proteins confer six $\mathrm{K}$ or $\mathrm{R}$ basic residues in the same region 65-87aa which exceed five basic residues reported to be needed for efficient amphipathic helix formation (Gibbs et al., 2003). Changing the Q69 and H75 of HK97 PB1-F2 to L increases mitochondrial localization of protein suggesting the involvement of this particular residue in MTS (Chen et al., 2010). Notably, mutation of conservative L in positions
72 and 77 did not abolish mitochondrial localization (Gibbs et al., 2003) in the presence of L69 and R75, thus while Gibs et al. showed that even loss of three $\mathrm{L}$ in position 72,75 , and 77 do not lead to the loss of mitochondrial localization, Chen et al. (2010) presented that both L in position 69 and 75 are needed for increased mitochondrial direction. It is probable that basic $\mathrm{R}$ in position 75 partially compensates L function in this site.

\section{PB1-F2 serine 35 and threonine 27 phosphorylation acceptor sites and their role in apoptosis}

Identification of the MTS in PB1-F2 and co-localization with mitochondria (Gibbs et al., 2003; Chen et al., 2001; Yamada et al., 2004) lead researchers inevitably to the assumption that PB1-F2 is connected to programmed cell death pathways. This was later enhanced by identification of interaction of PB1-F2 with voltage dependent anion channel (VDAC1), adenosine nucleotide transporter (ANT3), proteins of outer and inner mitochondrial membrane (Zamarin et al., 2005) as well as the intrinsic ability of PB1-F2 to create pores in membranes (Chanturiya et al., 2004). Unfortunately, it is still not known which particular residue of $\mathrm{PB} 1-\mathrm{F} 2$ are responsible for interaction with VDAC1 and ANT3 which could bring some light to understanding the importance of this relation. Proapoptotic effect was observed in cell dependent manner with obvious preferences for immune competent cells (Coleman, 2007). It has been shown more recently that proapoptosis is not general property of the PB1-F2 but is strain dependent particularly for commonly used laboratory strain PR8 and 1918 viruses (McAuley et al., 2010a). A necessary condition for proapoptotic function of PB1-F2 appears to be mitochondrial localization, thus it is not surprising that isolates with altered MTS crucial residues L69, L72, L/R75, K78 and L82 (Gibbs et al., 2003; Chen et al., 2010; Yamada et al., 2004) are not able to effectively induce cell death (McAuley et al., 2010a). Another factor affecting caspase 3 activation has been reported for PB1-F2 molecule. Extensive study identified the phosphorylation site of PB1F2 serine residue in position 35 as preferential. Negligible phosphorylation of the threonine 27 is due to closely localized proline residue in position 28 , which caused spatial hindrance to phosphorylation. Interaction of the PB1-F2 with protein kinase $\mathrm{C}$ alpha (PKCa) by yeast two hybrid system has also been proven. Additionally, while inhibitors of the PKCa (bisindolylmaleimide, staurosporine) decreased overall in the amount of phosphorylated PB1-F2, activators of PKCa (phorbol 12-myristate 13-acetate) increased phosphorylation two fold. Serine 35 mutant virus exhibited slightly impaired propagation in comparison with WT PB1-F2 virus on A549 or MDCK cells. However later, replication on human primary monocytes 36HPI revealed the decreased 
(a)

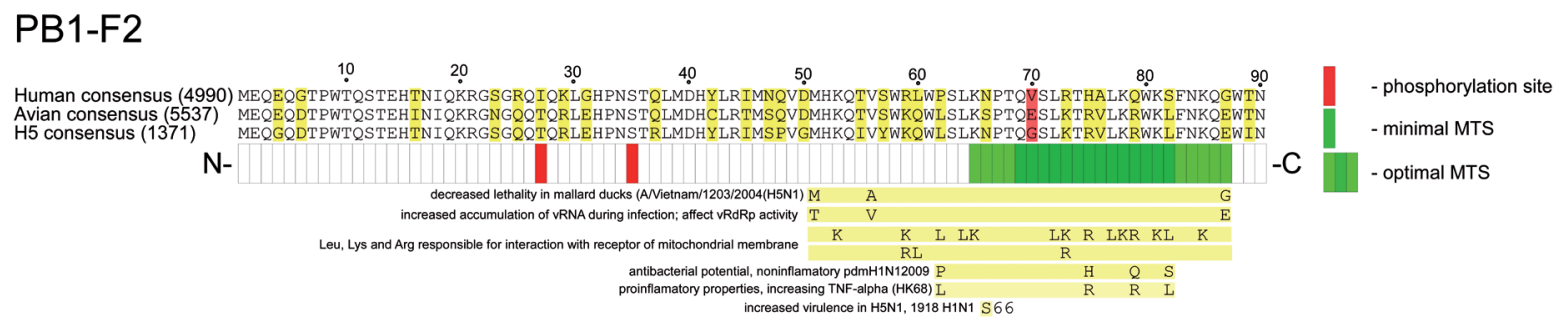

(b)

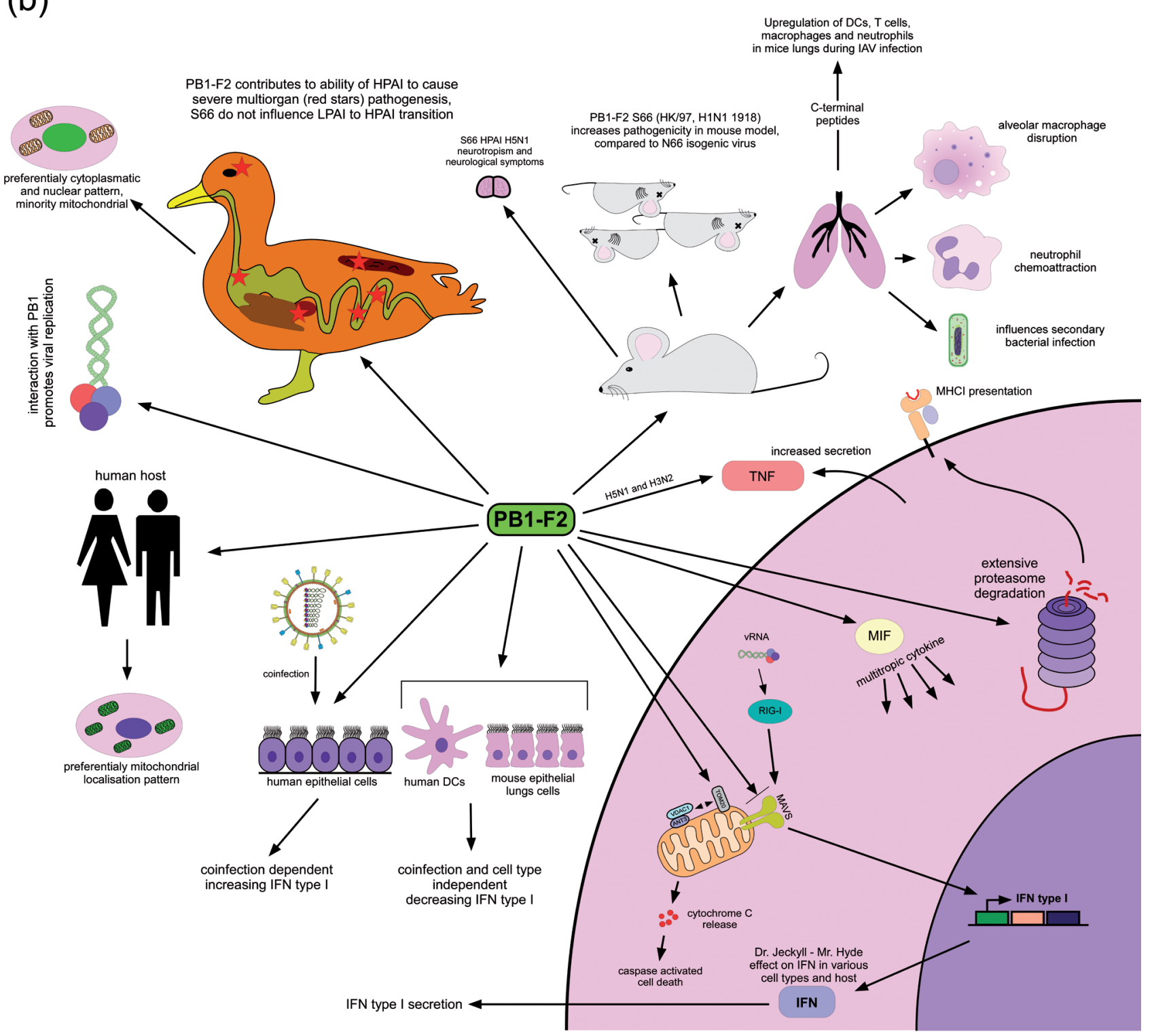

Fig. 2

Structural-functional map summarizing the complex behaviour of the PB1-F2

a) Alignment of the PB1-F2 consensus aminoacid sequences of human, avian and avian H5 subtype isolates representing described to date functionally important and most conserved aa residues created on www.fludb.org. Unhighlighted fields represent identical aa in given position, bright yellow represents non-identical aa in one consensus sequence and bright red represents various aa for all consensus sequences. b) Documented PB1-F2 impact on different cellular and viral protein functions, cell and host species type. 
growth of phosphorylation deficient mutant PB1-F2 S35L virus independently of IFN-I induction measured by MxA level. Importantly, WT virus possessing S35 PB1-F2 induces dramatically higher activity of apoptotic marker caspase 3 (Mitzner et al., 2009).

\section{Conclusion}

PB1-F2 protein differs significantly in aminoacid sequence as well as in almost all described functions, features and relations depending on strain, cell type and host species (Fig. 2). The significance of this protein is thus an enigma, although the high degree of presence of the PB1-F2 ORF in avian isolates and preservation of more than half a molecule (57aa) in swine and human isolates highly support the role of this protein for these hosts at least. Sequential variance resembling functional variability makes PB1-F2 a very versatile tool offering virus diverse opportunity to survive in different biological environments. Such functional complexity could serve as adaptation-evolutionary "punch" keeping IAV a continual danger to human health.

Acknowledgement. This work was supported by grants from the Slovak Research and Development Agency under the contract No. APVV-0250-10, No. DO7RP-0025-10 and by grants from the Scientific Grant Agency of the Ministry of Education of the Slovak Republic and Slovak Academy of Sciences VEGA 2/0176/12, 2/0100/13, and 2/0117/11.

\section{References}

Alymova IV, Green AM, van de Velde N, McAuley JL, Boyd KL, Ghoneim HE, McCullers JA, J. Virol. 85, 12324-12333, 2011. http://dx.doi.org/10.1128/JVI.05872-11

Boon AC, deBeauchamp J, Hollmann A, Luke J, Kotb M, Rowe S, Finkelstein D, Neale G, Lu L, Williams RW, Webby, RJ, J. Virol. 83, 10417-10426. http://dx.doi.org/10.1128/ IVI.00514-09

Coleman JR, Virol. J. 4, 9, 2007. http://dx.doi.org/10.1186/1743$\underline{422 X-4-9}$

Conenello GM, Tisoncik JR, Rosenzweig E, Varga ZT, Palese P, Katze MG, J. Virol. 85, 652-662, 2011. http://dx.doi. org/10.1128/JVI.01987-10

Conenello GM, Zamarin D, Perrone LA, Tumpey T, Palese P, PLoS Pathog. 3, 1414-1421, 2007. http://dx.doi.org/10.1371/ journal.ppat.0030141

Dudek SE, Wixler L, Nordhoff C, Nordmann A, Anhlan D, Wixler V, Ludwig S, Bio.l Chem. 392, 1135-1144, 2011. http:// dx.doi.org/10.1515/BC.2011.174

Ehrhardt C, Seyer R, Hrincius ER, Eierhoff T, Wolff T, Ludwig S, Microbes Infect. 12, 81-87, 2010. http://dx.doi.org/10.1016/j. micinf.2009.09.007
Fouchier R, Osterhaus AB, Steinbruner J, Yuen KY, Henderson DA, Klotz L, Sylvester E, Taubenberger JK, Ebright RH, Heymann DL, Nature 481, 257-259, 2012. http://dx.doi. org/10.1038/481443a

Friesenhagen J, Boergeling Y, Hrincius E, Ludwig S, Roth J, Viemann D, J. Leukoc. Biol. 92, 11-20, 2012. http://dx.doi. org/10.1189/jlb.0911479

Gibbs JS, Malide D, Hornung F, Bennink JR, Yewdell JW, J. Virol. 77, 7214-7224. http://dx.doi.org/10.1128/JVI.77.13.7214$\underline{7224.2003}$

Graef KM, Vreede FT, Lau YF, McCall AW, Carr SM, Subbarao K, Fodor E, J. Virol. 84, 8433-8445, 2003. http://dx.doi. org/10.1128/JVI.00879-10

Guan ZH, Zhang ML, Hou PL, Duan M, Cui YM, Wang XR, Acta Virol. 56, 199-207, 2012. http://dx.doi.org/10.4149/ av $2012 \quad 03 \quad 199$

Guillot L, Le Goffic R, Bloch S, Escriou N, Akira S, Chignard M, Si-Tahar, M, J. Biol. Chem. 280, 5571-5580, 2005. http:// dx.doi.org/10.1074/jbc.M410592200

Hai R, Schmolke M, Varga ZT, Manicassamy B, Wang TT, Belser JA, Pearce MB, Garcia-Sastre A, Tumpey TM, Palese P, J. Virol. 84, 4442-4450, 2010. http://dx.doi.org/10.1128/ JVI.02717-09

Hatta M, Gao P, Halfmann P, Kawaoka Y, Science 293, 1840-1842, 2001. http://dx.doi.org/10.1126/science.1062882

Hoper D, Kalthoff D, Hoffmann B, Beer M, J. Virol. 86, 1394-1404, 2012. http://dx.doi.org/10.1128/JVI.00797-11

Chanturiya AN, Basanez G, Schubert U, Henklein P, Yewdell JW, Zimmerberg J, J. Virol. 78, 6304-6312, 2004. http://dx.doi. org/10.1128/JVI.78.12.6304-6312.2004

Chen CJ, Chen GW, Wang CH, Huang CH, Wang YC, Shih SR, J. Virol. 84, 10051-10062, 2010. http://dx.doi.org/10.1128/ JVI.00592-10

Chen W, Calvo PA, Malide D, Gibbs J, Schubert U, Bacik I, Basta S, O'Neill R, Schickli J, Palese P, Henklein P, Bennink JR, Yewdell JW, Nat. Med. 7, 1306-13012, 2001. http://dx.doi. org/10.1038/nm1201-1306

Chevalier C, Al Bazzal A, VidicJ, Fevrier V, Bourdieu C, Bouguyon E, Le Goffic R, Vautherot JF, Bernard J, Moudjou M, Noinville S, Chich JF, Da Costa B, Rezaei H, Delmas, B, J. Biol. Chem. 285, 13233-13243, 2010. http://dx.doi. org/10.1074/jbc.M109.067710

Ichinohe T, Pang IK, Iwasaki, A, Nat. Immunol. 11, 404-410, 2010. http://dx.doi.org/10.1038/ni.1861

Iwai A, Shiozaki T, Kawai T, Akira S, Kawaoka Y, Takada A, Kida H, Miyazaki T, J. Biol. Chem. 285, 32064-32074, 2010. http://dx.doi.org/10.1074/jbc.M110.112458

Kido H, Okumura Y, Takahashi E, Pan HY, Wang S, Yao D, Yao M, Chida J, Yano M, Biochim. Biophys. Acta 1824, 186-194, 2012. http://dx.doi.org/10.1016/j.bbapap.2011.07.001

Kosik I, Krejnusova I, Bystricka M, Polakova K, Russ G, Acta Virol. 55, 45-53, 2011. http://dx.doi.org/10.4149/ av 20110145

Kosik I, Krejnusova I, Praznovska M, Polakova K, Russ G, Arch. Virol. 157, 811-817, 2012. http://dx.doi.org/10.1007/ $\underline{\text { s00705-012-1238-6 }}$

Le Goffic R, Bouguyon E, Chevalier C, Vidic J, Da Costa B, Leymarie O, Bourdieu C, Decamps L, Dhorne-Pollet S, Del- 
mas B, J. Immunol. 185, 4812-4823, 2010. http://dx.doi. org/10.4049/jimmunol.0903952

Le Goffic R, Leymarie O, Chevalier C, Rebours E, Da Costa B, Vidic J, Descamps D, Sallenave JM, Rauch M, Samson M, Delmas B, PLoS Pathog. 7, e1002202, 2011. http://dx.doi. org/10.1371/journal.ppat.1002202

Le Goffic R, Pothlichet J, Vitour D, Fujita T, Meurs E, Chignard M, Si-Tahar M, J. Immunol. 178, 3368-3372, 2007.

Marjuki H, Scholtissek C, Franks J, Negovetich NJ, Aldridge JR, Salomon R, Finkelstein D, Webster RG, Arch. Virol. 155, 925-934, 2010. http://dx.doi.org/10.1007/s00705010-0666-4

Mazur I, Anhlan D, Mitzner D, Wixler L, Schubert U, Ludwig S, Cell Microbiol. 10, 1140-1152, 2008. http://dx.doi. org/10.1111/j.1462-5822.2008.01116.x

McAuley JL, Hornung F, Boyd KL, Smith AM, McKeon R, Bennink J, Yewdell JW, McCullers JA, Cell Host Microbe 2, 240-249, 2007. http://dx.doi.org/10.1016/j.chom.2007.09.001

McAuley JL, Chipuk JE, Boyd KL, Van De Velde N, Green DR, McCullers JA, PLoS Pathog. 6, e1001014, 2010a. http:// dx.doi.org/10.1371/journal.ppat.1001014

McAuley JL, Zhang K, McCullers JA, J. Virol. 84, 558-564, $2010 \mathrm{~b}$. http://dx.doi.org/10.1128/JVI.01785-09

Meunier I, von Messling V, J. Virol. 86, 4271-4278, 2012. http:// dx.doi.org/10.1128/JVI.07243-11

Miao H, Hollenbaugh JA, Zand MS, Holden-Wiltse J, Mosmann TR, Perelson AS, Wu H, Topham DJ, J. Virol. 84, 6687-6698, 2010. http://dx.doi.org/10.1128/JVI.00266-10

Mitchell RA, Liao H, Chesney J, Fingerle-Rowson G, Baugh J, David J, Bucala, R, Proc. Natl. Acad. Sci. USA 99, 345-350, 2002. http://dx.doi.org/10.1073/pnas.012511599

Mitzner D, Dudek SE, Studtrucker N, Anhlan D, Mazur I, Wissing J, Jansch L, Wixler L, Bruns K, Sharma A, Wray V, Henklein P, Ludwig S, Schubert U, Cell Microbiol. 11, 1502-1516, 2009. http://dx.doi.org/10.1111/j.14625822.2009.01343.x

Moszynski P, BMJ 345, e4521, 2012. http://dx.doi.org/10.1136/ bmj.e4521

Muramoto Y, Noda T, Kawakami E, Akkina R, Kawaoka, Y, J. Virol. 87, 2455-2462, 2012.
Ozawa M, Basnet S, Burley, LM, Neumann G, Hatta M, Kawaoka Y, J. Virol. 85, 4596-4601, 2011. http://dx.doi.org/10.1128/ JVI.00029-11

Pena L, Vincent AL, Loving CL, Henningson JN, Lager KM, Li W, Perez DR, J. Gen. Virol. 93, 2204-2214, 2012a. http:// dx.doi.org/10.1099/vir.0.045005-0

Pena L, Vincent AL, Loving CL, Henningson JN, Lager KM, Lorusso A, Perez DR, J. Virol. 86, 5523-5532, 2012b. http://dx.doi. org/10.1128/JVI.00134-12

Pfanner N, Curr. Biol. 10, R412-5, 2000. http://dx.doi.org/10.1016/ S0960-9822(00)00507-8

Schmolke M, Manicassamy B, Pena L, Sutton T, Hai R, Varga ZT, Hale BG, Steel J, Perez DR, Garcia-Sastre A, PLoS Pathog. 7, e1002186, 2011. http://dx.doi.org/10.1371/journal. ppat. 1002186

Schnitzler SU, Schnitzler P, Virus Genes 39, 279-292, 2009. http:// dx.doi.org/10.1007/s11262-009-0404-8

Smith AM, Adler FR, McAuley JL, Gutenkunst RN, Ribeiro RM, McCullers JA, Perelson AS, PLoS Comput. Biol. 7, e1001081, 2011. http://dx.doi.org/10.1371/journal.pcbi.1001081

Solbak SM, Sharma A, Bruns K, Roder R, Mitzner D, Hahn F, Niebert R, Vedeler A, Henklein P, Schubert U, Wray V, Fossen T, Biochim. Biophys. Acta 1834, 568-582, 2012.

Ueda M, Yamate M, Du A, Daidoji T, Okuno Y, Ikuta K, Nakaya T, Virus Res. 136, 91-97, 2008. http://dx.doi.org/10.1016/j. virusres.2008.04.028

Varga ZT, Ramos I, Hai R, Schmolke M, Garcia-Sastre A, Fernandez-Sesma A, Palese P, PLoS Pathog. 7, e1002067, 2011. http://dx.doi.org/10.1371/journal.ppat.1002067

Wise HM, Foeglein A, Sun J, Dalton RM, Patel S, Howard W, Anderson EC, Barclay WS, Digard P, J. Virol. 83, 8021-8031, 2009. http://dx.doi.org/10.1128/JVI.00826-09

Yamada H, Chounan R, Higashi Y, Kurihara N, Kido H, FEBS Lett. 578, 331-336, 2004. http://dx.doi.org/10.1016/j. febslet.2004.11.017

Zamarin D, Garcia-Sastre A, Xiao X, Wang R, Palese, P, PLoS Pathog. 1, e4, 2005. http://dx.doi.org/10.1371/journal. ppat.0010004

Zamarin D, Ortigoza MB, Palese P, J. Virol. 80, 7976-7983, 2006. http://dx.doi.org/10.1128/JVI.00415-06 